\title{
Spaces of continuous and bounded functions over the field of geometric complex numbers
}

\section{Zafer Cakir}

\section{"Correspondence:}

zafer@gumushane.edu.tr

Department of Mathematical

Engineering, Gümüşhane University,

Gümüşhane, 29100, Turkey

\begin{abstract}
Following Grossman and Katz (Non-Newtonian Calculus, 1972), we construct the sets $B(A)$ and $C(A)$ of geometric complex-valued bounded and continuous functions, where $A$ denotes the compact subset of the complex plane $\mathbb{C}$. We show that the sets $B(A)$ and $C(A)$ of complex-valued bounded and continuous functions form a vector space with respect to the addition and scalar multiplication in the sense of multiplicative calculus. Finally, we prove that $B(A)$ and $C(A)$ are complete metric spaces.
\end{abstract}

MSC: 26A06; 11U10;08A05

Keywords: non-Newtonian calculus; algebraic structures with respect to non-Newtonian calculus; non-Newtonian function space

\section{Introduction}

Grossman and Katz [1], introduced the non-Newtonian calculus consisting of the branches of geometric, anageometric and biogeometric calculus, etc. Bashirov et al. [2] gave results with applications to the well-known properties of derivative and integral in the multiplicative calculus. Uzer [3] extended the multiplicative calculus to the complexvalued functions, was interested in the statements of some fundamental theorems and concepts of multiplicative complex calculus, and demonstrated some analogies between the multiplicative complex calculus and the classical calculus by theoretical and numerical examples. Recently, Çakmak and Başar [4] introduced the field $\mathbb{R}(N)$ of nonNewtonian real numbers and gave the triangle and Minkowski's inequalities in the sense of non-Newtonian calculus. They defined the complete metric spaces $\omega(N), \ell_{\infty}(N)$, $c(N), c_{0}(N)$ and $\ell_{p}(N)$ of all bounded, convergent, null and $p$-absolutely summable sequences in the sense of non-Newtonian calculus over the field $\mathbb{R}(N)$. Quite recently, Tekin and Başar [5] have introduced the spaces $\omega^{*}, \ell_{\infty}^{*}, c^{*}, c_{0}^{*}$ and $\ell_{p}^{*}$ over the nonNewtonian complex field $\mathbb{C}^{*}$ and obtained the corresponding results for these spaces, where $p \ddot{>0}$.

Following Bashirov et al. [2, 6, 7] and Uzer [3], Türkmen and Başar [8] obtained corresponding results for multiplicative complex numbers and the concept of multiplicative metric.

Following [8], the main purpose of this paper is the investigation of the space of functions defined by the multiplicative calculus. Following Türkmen and Başar [8], first we define the set $\mathbb{C}(G)$ of multiplicative complex numbers by

2013 Cakir licensee Springer. This is an Open Access article distributed under the terms of the Creative Commons Attribution License (http://creativecommons.org/licenses/by/2.0), which permits unrestricted use, distribution, and reproduction in any medium, provided the original work is properly cited. 


$$
\begin{aligned}
\mathbb{C}(G) & :=\left\{w=u \oplus i_{g} \odot v: u, v \in \mathbb{R}(G) \text { and } i_{g}=\sqrt{1 \ominus e^{G}}\right\} \\
& =\left\{e^{z}=e^{x} \cdot\left(e^{y}\right)^{\ln e^{i}}: e^{x}, e^{y} \in \mathbb{R}(G) \text { and } e^{i}=e^{\sqrt{-1}}\right\} \\
& =\left\{e^{x+i y}: x \in \mathbb{R} ;-\pi<y \leq \pi \text { and } i=\sqrt{-1}\right\} \\
& =\left\{e^{z}: z \in \mathbb{C}_{\mathrm{str}}\right\},
\end{aligned}
$$

where $\mathbb{R}(G)$ denotes the set of multiplicative real numbers and

$$
\mathbb{C}_{\text {str }}:=\{z=x+i y: x \in \mathbb{R} ;-\pi<y \leq \pi\}
$$

It is easy to see that $\mathbb{C}(G)=\mathbb{C} \backslash\{0\}$. It is clear from the definition of complex exp function that $\alpha(z)=e^{z} \neq 0$ for all $z \in \mathbb{C}_{\text {str }}$. Since $\alpha$-generator is a bijective function, it maps all complex numbers without zero to the set of values.

We suppose throughout that the $A$ is a compact subset of the complex plane $\mathbb{C}$ and $(\mathbb{C}(G), \oplus, \odot)$ denotes the geometric complex field introduced by Türkmen and Başar [8].

We will consider the sets $B(A)$ and $C(A)$ in the following forms:

$$
\begin{aligned}
& B(A):=\left\{f:\left.A \rightarrow \mathbb{C}(G)\left|\exists K \in \mathbb{R}^{+} \ni \forall x \in A,\right| f(x)\right|_{G} \leq K\right\}, \\
& C(A):=\{f: A \rightarrow \mathbb{C}(G) \mid f \text { is continuous on } A\} .
\end{aligned}
$$

For $f, g \in B(A)$ and $\lambda \in \mathbb{C}(G)$, we define the operations addition $(\boxplus)$ and scalar multiplication $(\square)$ by

$$
\begin{aligned}
\boxplus: \quad B(A) \times B(A) & \longrightarrow \mathbb{C}(G) \\
(f, g) & \longrightarrow(f \boxplus g)(x)=f(x) \oplus g(x), \\
\square: \mathbb{C}(G) \times B(A) & \longrightarrow \mathbb{C}(G) \\
(\lambda, f) & \longrightarrow(\lambda \boxminus f)(x)=\lambda \odot f(x) .
\end{aligned}
$$

\section{Multiplicative complex field and related properties}

Theorem 2.1 The set $B(A)$ is a vector space with respect to the algebraic operations addition $(\boxplus)$ and scalar multiplication $(\square)$.

Proof Let $x \in A, f, g \in B(A)$ and $\lambda \in \mathbb{C}(G)$. Then, since $f, g \in B(A)$, there exist positive numbers $K_{1}$ and $K_{2}$ such that $|f(x)|_{G} \leq K_{1}$ and $|g(x)|_{G} \leq K_{2}$ for all $x \in A$. Therefore, one can see by the triangle inequality that

$$
\begin{aligned}
|(f \boxplus g)(x)|_{G} & =|f(x) \oplus g(x)|_{G} \leq|f(x)|_{G} \oplus|g(x)|_{G} \\
& \leq K_{1} K_{2}=K ; \quad K \in \mathbb{R}^{+} .
\end{aligned}
$$

This means that $f \boxplus g \in B(A)$.

Since the equality $\left|\alpha_{1} \odot \alpha_{2}\right|_{G}=\left|\alpha_{1}\right|_{G} \odot\left|\alpha_{2}\right|_{G}$ holds for $\alpha_{1}, \alpha_{2} \in \mathbb{C}(G)$, by using this fact, we observe that

$$
\begin{aligned}
|(\lambda \odot f)(x)|_{G} & =|\lambda \odot f(x)|_{G}=|\lambda|_{G} \odot|f(x)|_{G} \\
& =\left[|f(x)|_{G}\right]^{\ln |\lambda|_{G}}<\infty .
\end{aligned}
$$

That is, $\lambda \boxminus f \in B(A)$. 
(V1) Addition is commutative, that is,

$$
\begin{aligned}
(f \boxplus g)(x) & =f(x) \oplus g(x) \\
& =e^{\ln f(x)+\ln g(x)} \\
& =e^{\ln g(x)+\ln f(x)} \\
& =g(x) \oplus f(x) \\
& =(g \boxplus f)(x) .
\end{aligned}
$$

(V2) Addition is associative, i.e.,

$$
\begin{aligned}
{[(f \boxplus g) \boxplus h](x) } & =[f(x) \oplus g(x)] \oplus h(x) \\
& =e^{[\ln f(x)+\ln g(x)]+\ln h(x)} \\
& =e^{\ln f(x)+[\ln g(x)+\ln h(x)]} \\
& =f(x) \oplus[g(x) \oplus h(x)] \\
& =[f \boxplus(g \boxplus h)](x) .
\end{aligned}
$$

(V3) An identity element exists for addition. Indeed, since

$$
\begin{aligned}
& {[f \boxplus 0](x)=f(x)} \\
& \quad \Longrightarrow \quad f(x) \oplus \dot{0}=f(x) \\
& \quad \Longrightarrow \quad e^{\ln f(x)} e^{\ln \dot{0}}=f(x) \\
& \quad \Longrightarrow \quad f(x) \cdot \dot{0}=f(x) \\
& \quad \Longrightarrow \quad \dot{0}=e^{0}=1 \in B(A),
\end{aligned}
$$

the identity element is the function 0 such that $0(x)=1$ for all $x \in A$.

(V4) The inverse element of any $f \in B(A)$ exists such that

$$
(f \boxplus g)(x)=f(x) \oplus g(x)=e^{\ln f(x)} e^{\ln g(x)}=f(x) \cdot g(x)=1,
$$

which yields that

$$
g(x)=\frac{1}{f(x)} \quad \text { for all } f \in B(A),
$$

i.e., the inverse element of $f \in B(A)$ with respect to $\boxplus$ is $g=1 / f$.

(V5) Scalar multiplication distributes to the addition over the field. Indeed, since

$$
\begin{aligned}
{[(\lambda \oplus \mu) \boxminus f](x) } & =(\lambda \oplus \mu) \odot f(x)=e^{(\ln \lambda+\ln \mu) \ln f(x)}=e^{\ln \lambda \ln f(x)+\ln \mu \ln f(x)} \\
& =[\lambda \odot f(x)] \oplus[\mu \odot f(x)] \\
& =(\lambda \odot f)(x) \boxplus(\mu \odot f)(x),
\end{aligned}
$$

scalar multiplication distributes to the addition over the field. 
(V6) Scalar multiplication distributes to vector addition, i.e.,

$$
\begin{aligned}
{[\lambda \odot(f \boxplus g)](x) } & =\lambda \odot[f(x) \oplus g(x)] \\
& =e^{\ln \lambda \cdot \ln f(x)+\ln g(x)]} \\
& =e^{\ln \lambda \cdot \ln f(x)+\ln \lambda \cdot \ln g(x)} \\
& =[\lambda \odot f(x)] \oplus[\lambda \odot g(x)] \\
& =(\lambda \odot f)(x) \boxplus(\lambda \odot g)(x) .
\end{aligned}
$$

(V7) Compatibility of scalar multiplication with field multiplication holds:

$$
\begin{aligned}
{[(\lambda \odot \mu) \odot f](x) } & =(\lambda \odot \mu) \odot f(x) \\
& =e^{\ln \lambda \cdot \ln \mu \cdot \ln f(x)} \\
& =e^{\ln \lambda \cdot(\ln \mu \cdot \ln f(x))} \\
& =\lambda \odot[\mu \odot f(x)] \\
& =[\lambda \odot(\mu \odot f)](x) .
\end{aligned}
$$

(V8) $e$ is the identity element of scalar multiplication. It is easy to see that

$$
(e \odot f)(x)=e \odot f(x)=e^{\ln e \ln f(x)}=f(x),
$$

which says that the identity element of scalar multiplication is $e$.

From (V1)-(V8) vector space axioms are satisfied. Hence $B(A)$ is a vector space over $\mathbb{C}(G)$ with the algebraic operations addition $(\boxplus)$ and scalar multiplication $(\square)$.

Theorem 2.2 The set $C(A)$ is a subspace of the space $B(A)$ with addition $(\boxplus)$ and scalar multiplication $(\square)$.

Proof First, we should show that $C(A) \neq \emptyset$ and $C(A) \subset B(A)$.

Since $0(x)=1$ for all $x \in A, 0 \in C(A)$, that is, the set $C(A)$ is not empty.

Suppose that $C(A) \not \subset B(A)$. Then there is $f \in C(A)$ such that $\left|f\left(x_{n}\right)\right|_{G} \geq n$ for $x_{n} \in A$ for all $n \in \mathbb{N}$. Since $A$ is compact, $\left(x_{n}\right)$ is a bounded geometric sequence. So, $\left(x_{n}\right)$ has at least one convergent subsequence $\left(x_{n_{k}}\right)$, say $x_{n_{k}} \stackrel{G}{\rightarrow} x_{0}$, as $k \rightarrow \infty$. Since $A$ is closed $x_{0} \in A$, hence $f$ is continuous at the point $x_{0}$. Therefore, for $\epsilon>1$, there exists at least $\delta>1$ such that $\left|f(x) \ominus f\left(x_{0}\right)\right|_{G}<\epsilon$ for all $\left|x \ominus x_{0}\right|_{G}<\delta$. Now, we choose $\epsilon=e$. Thus, we have $|f(x)|-$ $\left|f\left(x_{0}\right)\right|_{G} \leq\left|f(x) \ominus f\left(x_{0}\right)\right|_{G}<\epsilon$ which leads to $|f(x)|_{G}<e+\left|f\left(x_{0}\right)\right|_{G}$. This contradicts the fact $\left|f\left(x_{n}\right)\right|_{G} \geq n$. Hence, $f \in B(A)$ and the inclusion $C(A) \subset B(A)$ holds.

Let $x \in A, f, g \in C(A)$ and $\lambda, \mu \in \mathbb{C}(G)$. Then we have

$$
\begin{aligned}
{[(\lambda \odot f) \boxplus(\mu \odot g)](x) } & =[\lambda \odot f(x)] \oplus[\mu \odot g(x)]=e^{\ln \lambda \cdot \ln f(x)+\ln \mu \cdot \ln g(x)} \\
& =\left[e^{\ln f(x)}\right]^{\ln \lambda}\left[e^{\ln g(x)}\right]^{\ln \mu} \\
& =[f(x)]^{\ln \lambda}[g(x)]^{\ln \mu} \in C(A) .
\end{aligned}
$$

Therefore, the algebraic operations $\boxplus$ and $\square$ are closed on $C(A)$. 
Axioms (V1)-(V8) on $C(A)$ can be fulfilled in the same way as in the proof of Theorem 2.1.

\section{Geometric metric spaces}

For each $f, g \in B(A)$, we define $d_{G}$ by

$$
\begin{aligned}
d_{G}: B(A) \times B(A) & \longrightarrow \mathbb{R}^{+}(G) \\
(f, g) & \longrightarrow d_{G}(f, g)=\left.\sup _{x \in A} f(x) \ominus g(x)\right|_{G} .
\end{aligned}
$$

Theorem 3.1 $\left(B(A), d_{G}\right)$ is a complete metric space.

Proof Let $x \in A$ and $f, g, h \in B(A)$. Now, we check the metric axioms. Let $e^{f_{1}(y)}=f(x), e^{g_{1}(y)}=$ $g(x), e^{h_{1}(y)}=h(x) \in B(A)$ for $x, y \in A$, where $f_{1}, g_{1}$ and $h_{1}$ are the complex-valued bounded functions.

(GM1) Non-negative property holds: $\dot{0}=1, \forall f, g \in B(A) \Longrightarrow d_{G}(f, g) \geq \dot{0}$.

$(\mathrm{GM} 2) d_{G}(f, g)=\dot{0} \Longleftrightarrow f(x) \ominus g(x)=\dot{0}$.

$\Rightarrow$ : From the definition of supremum, we can write

$$
\begin{aligned}
d_{G}(f, g) & =1 \\
\Longrightarrow & \sup _{x \in A}|f(x) \ominus g(x)|_{G}=1 \\
\Longrightarrow & |f(x) \ominus g(x)|_{G} \leq 1,
\end{aligned}
$$

and from the definition of geometric absolute value (see [8]),

$$
|f(x) \ominus g(x)|_{G} \geq 1=e^{0} .
$$

Using (3.2) and (3.3), we have

$$
\begin{aligned}
& |f(x) \ominus g(x)|_{G}=1 \\
& \Longrightarrow\left|\frac{f(x)}{g(x)}\right|_{G}=1 \\
& \Longrightarrow\left|\frac{e^{f_{1}(y)}}{e^{g_{1}(y)}}\right|_{G}=1 \\
& \Longrightarrow\left|e^{f_{1}(y)-g_{1}(y)}\right|_{G}=1 \\
& \Longrightarrow e^{\left|f_{1}(y)-g_{1}(y)\right|}=1=e^{0} \\
& \Longrightarrow\left|f_{1}(y)-g_{1}(y)\right|=0 \\
& \Longrightarrow f_{1}(y)-g_{1}(y)=0 \\
& \Longrightarrow f_{1}(y)=g_{1}(y) \\
& \Longrightarrow e^{f_{1}(y)}=e^{g_{1}(y)} \\
& \Longrightarrow f(x)=g(x) .
\end{aligned}
$$


$\Leftarrow$ : Conversely, we get

$$
\begin{aligned}
f(x) & =g(x) \\
& \Longrightarrow\left|\frac{f(x)}{g(x)}\right|=1 \\
& \Longrightarrow|f(x) \ominus g(x)|_{G}=1 \\
& \Longrightarrow \sup _{x \in A}|f(x) \ominus g(x)|_{G}=1 \\
& \Longrightarrow d_{G}(f, g)=1 .
\end{aligned}
$$

(GM3) Symmetry property holds. From the definition of the relation $d_{G}$, we have

$$
\begin{aligned}
d_{G}(f, g) & =\sup _{x \in A}|f(x) \ominus g(x)|_{G} \\
& =\sup _{x \in A}\left|\frac{f(x)}{g(x)}\right|_{G}=\sup _{y \in A}\left|\frac{e^{f_{1}(y)}}{e^{g_{1}(y)}}\right|_{G} \\
& =\sup _{y \in A}\left|e^{f_{1}(y)-g_{1}(y)}\right|_{G}=\sup _{y \in A} e^{\left|f_{1}(y)-g_{1}(y)\right|} \\
& =\sup _{y \in A} e^{\left|g_{1}(y)-f_{1}(y)\right|}=\sup _{y \in A}\left|e^{g_{1}(y)-f_{1}(y)}\right|_{G} \\
& =\sup _{y \in A}\left|\frac{e^{g_{1}(y)}}{e^{f_{1}(y)}}\right|_{G}=\sup _{x \in A}\left|\frac{g(x)}{f(x)}\right|_{G} \\
& =\sup _{x \in A}|g(x) \ominus f(x)|_{G} \\
& =d_{G}(g, f) .
\end{aligned}
$$

(GM4) The triangle inequality holds. Firstly we will get

$$
\begin{aligned}
|f(x) \ominus g(x)|_{G} & =\left|\frac{f(x)}{g(x)}\right|_{G}=\left|\frac{e^{f_{1}(y)}}{e^{g_{1}(y)}}\right|_{G}=\left|e^{f_{1}(y)-g_{1}(y)}\right|_{G} \\
& =e^{\left|f_{1}(y)-g_{1}(y)\right|}=e^{\left|f_{1}(y)-h_{1}(y)+h_{1}(y)-g_{1}(y)\right|} \\
& \leq e^{\left|f_{1}(y)-h_{1}(y)\right|+\left|h_{1}(y)-g_{1}(y)\right|}=e^{\left|f_{1}(y)-h_{1}(y)\right|} \cdot e^{\left|h_{1}(y)-g_{1}(y)\right|} \\
& =\left|e^{f_{1}(y)-h_{1}(y)}\right|_{G} \cdot\left|e^{h_{1}(y)-g_{1}(y)}\right|_{G}=\left|\frac{e^{f_{1}(y)}}{e^{h_{1}(y)}}\right|_{G} \cdot\left|\frac{e^{h_{1}(y)}}{e^{g_{1}(y)}}\right|_{G} \\
& =\left|\frac{f(x)}{h(x)}\right|_{G} \cdot\left|\frac{h(x)}{g(x)}\right|_{G}=|f(x) \ominus h(x)|_{G} \oplus|h(x) \ominus g(x)|_{G} .
\end{aligned}
$$

Therefore, one can easily see that

$$
\begin{aligned}
\sup _{x \in A}|f(x) \ominus g(x)|_{G} & \leq \sup _{x \in A}\left[|f(x) \ominus h(x)|_{G} \oplus|h(x) \ominus g(x)|_{G}\right] \\
& \leq \sup _{x \in A}|f(x) \ominus h(x)|_{G} \oplus \sup _{x \in A}|h(x) \ominus g(x)|_{G},
\end{aligned}
$$


which leads us to the desired inequality

$$
d_{G}(f, g) \leq d_{G}(f, h) \oplus d_{G}(h, g) .
$$

Properties (GM1)-(GM4) imply that $\left(B(A), d_{G}\right)$ is a metric space.

Suppose that $\left(f_{n}\right)$ is a Cauchy sequence in the metric space $\left(B(A), d_{G}\right)$. Then, for every $\varepsilon>1$, there is an $n_{0}=n_{0}(\varepsilon)$ such that

$$
d_{G}\left(f_{n}, f_{m}\right)=\sup _{x \in A}\left|f_{n}(x) \ominus f_{m}(x)\right|_{G}<\varepsilon
$$

for all $m, n>n_{0}$. Hence, $\left\{f_{n}(x)\right\}$ is a Cauchy sequence of geometric complex numbers for each fixed $x \in A$. Since $\mathbb{C}(G)$ is complete by Theorem 4.5 of Türkmen and Başar [8], the sequence $\left\{f_{n}(x)\right\}$ is convergent, say $f_{n}(x) \stackrel{G}{\rightarrow} f(x)$ for $x \in A$, as $n \rightarrow \infty$. By letting $m \rightarrow \infty$ with $n>n_{0}$, we derive from (3.4) that $\sup _{x \in A}\left|f_{n}(x)-f(x)\right|_{G} \leq \epsilon$. Therefore we have $\mid f_{n}(x)-$ $\left.f(x)\right|_{G} \leq \epsilon$ for all $n>n_{0}$ and for all $x \in A$. That is to say, for every $\epsilon>1$, there exists at least $n_{0}=n_{0}(\epsilon) \in \mathbb{N}$ such that $\left|f_{n}(x)-f(x)\right|_{G} \leq \epsilon$ for all $n>n_{0}$ and for all $x \in A$. This means that the sequence $\left(f_{n}\right)$ converges uniformly to $f$ as $n \rightarrow \infty$.

Additionally, since there exists a $K>0$ such that

$$
|f(x)|_{G}=\left|f(x)-f_{n}(x)+f_{n}(x)\right|_{G} \leq\left|f(x)-f_{n}(x)\right|_{G} \oplus\left|f_{n}(x)\right|_{G} \leq \epsilon \cdot K
$$

for all $x \in A$ and for all $n \in \mathbb{N}, f \in B(A)$. That is to say, an arbitrary Cauchy sequence in the metric space $\left(B(A), d_{G}\right)$ is convergent. This completes the proof.

It is obvious that $d_{G}$ is an induced metric from the norm $\|\cdot\|_{G}$, that is,

$$
\|f\|_{G}=d_{G}(f, 0)=\sup _{x \in A}|f(x) \ominus 0(x)|_{G} ; \quad f \in B(A) .
$$

So, we have the following as a direct consequence of Theorem 3.1.

Corollary 3.2 $\left(B(A),\|\cdot\|_{G}\right)$ is a Banach space, where $\|\cdot\|_{G}$ is defined by (3.5).

Theorem $3.3\left(C(A), d_{G}^{\prime}\right)$ is a complete metric space, where $d_{G}^{\prime}$ is defined on the space $C(A)$ by

$$
\begin{aligned}
d_{G}^{\prime}: \quad C(A) \times C(A) & \longrightarrow \mathbb{R}^{+}(G) \\
(f, g) & \longrightarrow d_{G}^{\prime}(f, g)=\max _{x \in A}|f(x) \ominus g(x)|_{G} .
\end{aligned}
$$

Theorem 3.3 leads to the following result.

Corollary 3.4 $\left(C(A),\|\cdot\|_{G}\right)$ is a Banach space, where $\|\cdot\|_{G}$ is defined on the space $C(A)$ by

$$
\|f\|_{G}=d_{G}^{\prime}(f, 0)=\max _{x \in A}|f(x) \ominus 0(x)|_{G} ; \quad f \in C(A) .
$$




\section{Competing interests}

The author declares that he has no competing interests.

\section{Acknowledgements}

The author would like to thank Professor Feyzi Başar, Department of Mathematics, Faculty of Arts and Sciences, Fatih University, The Hadımköy Campus, Büyükçekmece, 34500-İstanbul, Turkey, for his careful reading and constructive criticism of an earlier version of this paper, which improved the presentation and its readability. The author is very grateful to the anonymous referee for many helpful suggestions and comments on the paper. Finally, the author should express his gratitude to Ph.D. students Sebiha Tekin and Ahmet Faruk Çakmak for their valuable help for revising the manuscript in the light of reviewer's suggestions. The main results of this paper were presented in part at the conference First International Conference on Analysis and Applied Mathematics (ICAAM 2012) held October 18-21, 2012 in Gümüşhane, Turkey at the University of Gümüşhane.

Received: 1 January 2013 Accepted: 19 July 2013 Published: 5 August 2013

\section{References}

1. Grossman, M, Katz, R: Non-Newtonian Calculus. Lee Press, Pigeon Cove (1972)

2. Bashirov, AE, Kurpınar, EM, Özyapıcı, A: Multiplicative calculus and its applications. J. Math. Anal. Appl. 337, 36-48 (2008)

3. Uzer, A: Multiplicative type complex calculus as an alternative to the classical calculus. Comput. Math. Appl. 60 2725-2737 (2010)

4. Çakmak, AF, Başar, F: On the classical sequence spaces and non-Newtonian calculus. J. Inequal. Appl. 2012, Article ID 932734 (2012)

5. Tekin, S, Başar, F: Certain sequence spaces over the non-Newtonian complex field. Abstr. Appl. Anal. 2013, Article ID 739319 (2013)

6. Bashirov, A, Rıza, M: On complex multiplicative differentiation. TWMS J. App. Eng. Math. 1(1), $75-85$ (2011)

7. Bashirov, AE, Mısırlı, E, Tandoğdu, Y, Özyapıcı, A: On modeling with multiplicative differential equations. Appl. Math. J. Chin. Univ. Ser. B 26(4), 425-438 (2011)

8. Türkmen, C, Başar, F: Some basic results on the sets of sequences with geometric calculus. Commun. Fac. Sci. Univ. Ank. Ser. A1 Math. Stat. 61(2), 17-34 (2012)

doi:10.1186/1029-242X-2013-363

Cite this article as: Cakir: Spaces of continuous and bounded functions over the field of geometric complex numbers. Journal of Inequalities and Applications 2013 2013:363.

\section{Submit your manuscript to a SpringerOpen ${ }^{\circ}$ journal and benefit from:}

- Convenient online submission

- Rigorous peer review

- Immediate publication on acceptance

Open access: articles freely available online

High visibility within the field

- Retaining the copyright to your article 